\title{
The form of the psychophysical function near threshold'
}

\author{
LAWRENCE E. MARKS AND JOSEPH C. STEVENS 2 \\ JOHN B. PIERCE FOUNDATION LABORATORY AND YALE UNIVERSITY
}

Psychophysical functions typically depart from a simple power law in the vicinity of the absolute threshold. Five versions of the psychophysical power law have been proposed to describe the function near threshold. An account is given of some of the difficulties encountered in attempts to decide among the various versions by means of empirical tests.

Approximately 15 years have passed since S. S. Stevens (1953) proposed the psychophysical power law, according to which subjective magnitude $\psi$ is related to stimulus magnitude $\varphi$ by a power function:

$$
\psi=k \varphi^{\beta}
$$

As the experimental evidence mounts to support this basic psychophysical relation, it is natural to inquire whether systematic deviations occur. Those who did early work in this area of psychophysics soon noted that the simple form of the power function stated in Eq. I fails to describe the growth of subjective magnitude near the absolute threshold. Plotted in $\log -\log$ coordinates the psychophysical function typically departs from linearity by turning down at the low end.

Some five modifications of Eq. 1 have been proposed to rectify the power function near threshold. Fagot and Stewart (1968) and Stewart, Fagot, and Eskildsen (1967) have tried to decide between two of these modifications on the basis of experimentation. Their articles prompt this brief review of the five modifications and some of the related issues.

\section{(1) Subtraction from $\varphi$}

One modification of the power law seems to have been formulated first by Ekman (1956) and later by Luce (1959) and S. S. Stevens (1959). This version of the power law (sometimes termed the $\varphi$ translation) states that

$$
\psi=\mathrm{k}\left(\varphi-\varphi_{0}\right)^{\beta}
$$

where $\varphi_{0}$ approximates threshold. In other words, when the stimulus is regarded as a distance re an effective threshold, the apparent departures from the power law may be eliminated. For example, apparent warmth and apparent cold turned out to be power functions when the stimulus was reckoned to be an increase (warmth) or decrease (cold) in temperature re "physiological zero" (approximately $305 \mathrm{deg} \mathrm{K}$ ) (J. C. Stevens \& S. S. Stevens, 1960).

(2) Addition to $\psi$

Another modification, sometimes called the $\psi$ translation, is

$$
\psi+\mathrm{k} \varphi_{0}^{\beta}=\mathrm{k} \varphi^{\beta}
$$

This translation modifies the power function along the psychological, rather than the physical, continuum.

Figure 1 shows graphical comparisons of the two kinds of translations, those represented by Eqs. 2 and 3. The graphical form of the functions in $\log$-log coordinates depends on the value of the exponent $\beta$. When $\beta=1$ both equations give the same graphical form.

The $\psi$ translation is based on the assumption that the deviation near threshold may be attributed to masking caused by intrinsic physiological noise or by noise deliberately introduced as a masker. (The suggestion that noise might cause a constant subtraction from loudness can be traced back to the 1930s; for a review, see S. S. Stevens, 1966.) Galanter and Messick (1961) compared Eqs. 2 and 3 for their ability to describe estimates of subjective loudness. Empirically, neither equation was clearly superior to the other (the predicted differences are probably too small), but the authors preferred the $\varphi$ translation for theoretical reasons. A mathematical equivalent of Eq. 3 was also used in 1961 by Ekman to rectify estimations of saltiness. The implied value of $\varphi_{0}$, however, was negative rather than positive; i.e., the psychophysical function, plotted in log-log coordinates, had a shape that was concave upward near threshold. Such a shape would seem contrary to an interpretation of the $\psi$ translation in terms of simple subtractive masking, which Fagot and Stewart (1968) applied to the brightness function and Lochner and Burger (1961) to the loudness function. However, the reader is referred to McBurney (1966) for a more recent and elaborate investigation of the form of the psychophysical function for taste.

In their review of the psychophysical function for loudness, Fagot and Stewart (1968) stated that McGill (1960) found the $\psi$ translation to be appropriate for individual loudness functions. This is not wholly correct. McGill pointed out that his Ss seemed to be making interval rather than magnitude (ratio) judgments. An


Fig. 1. Two modifications of the psychophysical power law: the $\psi$ translation $\left(\psi=k\left[\varphi^{\beta}-\varphi_{0}^{\beta}\right]\right)$, and the $\varphi$ translation $\left(\psi=k\left[\varphi-\varphi_{0}\right]^{\beta}\right)$. For all the functions the values of $\varphi_{0}$ and of $k$ were set equal to 1.0. Note that for $\beta=0.5$ the $\varphi$-translated function turns down more sharply near the threshold, whereas for $\beta=2.0$ the $\psi$-translated function turns down more sharply near the threshold. For $\beta=1.0$ the two functions would coincide. 
additive constant was necessary in order to transform the responses into a ratio scale. It is not clear, therefore, that the additive constant in. McGill's equation and the threshold constant in Eq. 3 play the same role.

\section{(3) Addition to $\psi$ and Addition to $\varphi$}

After first accepting the $\psi$ translation (Zwislocki \& Hellman, 1960), Zwislocki (1965) later abandoned the $\psi$ translation in favor of a more elaborate model for loudness. This third modification of the power law reads:

$$
\psi+\operatorname{kap}_{0}^{\beta}=k\left(\varphi+c_{0}\right)^{\beta}
$$

Eq. 4 suggests that intrinsic noise (related to the term $c_{0}$ ) adds to the stimulus power but at the same time subtracts from the apparent loudness by means of masking. The degree to which the intrinsic noise adds to the power of the stimulus and subtracts from the apparent loudness is said to relate to the size of the critical band. This conception of the power law makes some interesting predictions about the shape of the psychophysical power function as it relates to the size of $\beta$. When $\beta$ equals 1.0 , Eq. 4 reduces to Eq. 1, and when $\beta>1$ the psychophysical function should be concave upward when plotted in $\log -\log$ coordinates. To the best of our knowledge, these predictions have yet to be tested on a variety of sensory continua.

\section{(4) Subtraction from $\psi$ and Subtraction from $\varphi$}

A fourth modification of Eq. 1 was proposed by Mountcastle, Poggio, and Werner (1963) to describe the relation between frequency of firing in thalamic cells and degree of limb rotation. This modification can be written

$$
\downarrow-\mathrm{a}=\mathrm{k}\left(\varphi-\varphi_{0}\right)^{\beta}
$$

Eq. 5 is a hybrid of Eqs. 2 and 3. Accordingly, the stimulus is regarded as a change in physical level $\left(\varphi-\varphi_{0}\right)$, but a $\psi$ translation, due to the spontaneous activity (a), is also recognized. Although the present authors are not aware of any application of Eq. 5 to psychophysical data, it seems likely that it would be able to describe psychophysical data at least as well as, and in some instances better than, either Eq. 2 or 3 . Translation of the stimulus scale and of the sensation scale are not necessarily incompatible (see Fagot, 1966).

\section{(5) Bisegmentation}

A fifth modification of Eq. 1 was proposed for brightness by
Ekman (1967). Ekman regards the brightness function as possibly composed of two segments. The upper segment is a power function with $\beta$ equal to approximately 0.33 . The lower segment (near the threshold) was described by the equation

$$
\psi=\mathbf{k}\left(\varphi-\varphi_{0}\right)
$$

Ekman reported that the $\psi$ translation did not provide a very good description of his brightness data.

Ekman's result takes on added interest in the light of extensive evidence (S. S. Stevens, 1966; S. S. Stevens \& Guirao, 1967) that the psychophysical functions for brightness under simultaneous contrast and for loudness under masking are bisegmented. Ekman's equation may, therefore, be regarded as a special case of S. S. Stevens' principle of "power transformation." According to Stevens, the effects of contrast and masking are neither simply subtractive nor simply multiplicative, but involve a change in the exponent of the psychophysical power law. In fact, bisegmented functions would appear to provide excellent descriptions of most of the brightness data reported by Fagot and Stewart (1968).

\section{SOME COMMENTS ON THE $\varphi$ - AND $\psi$-TRANSLATED FUNCTIONS}

The modifications of the power law expressed in Eqs. 4-6, whatever their potential merit may be, have yet to be examined over a variety of sensory continua. Eq. 4 was tailored specifically to deal with loudness, although it may prove applicable to other continua in altered versions. Eq. 5 has been fitted to physiological data but apparently not as yet to psychophysical data. Eq. 6 has been used to describe the results of only one experiment on one sensory continuum. Eqs. 2 and 3, on the other hand, have been used in studies of several sensory continua. The comments that follow are consequently directed in the main toward an empirical comparison of the $\varphi$ - and $\psi$-translated functions.

The major obstacle to deciding empirically among the various alternatives is that the results predicted by different equations are usually rather similar, relative to our present ability to measure subjective magnitude. The problem is illustrated by Fig. 2 , which contrasts the $\psi$ - and $\varphi$-translated versions of the sone function relating loudness to sound pressure. The $\psi$ version has been drawn under the assumption that $\varphi_{0}=0.0002$ microbar. The $\varphi$ version has been drawn twice in Fig. 2, once under the assumption that $\varphi_{0}=0.0002$ microbar and once under the assumption that $\varphi_{0}=0.00035$ microbar. For all three functions $\beta=0.6$. Each function was drawn to pass through the point $40 \mathrm{dBSPL}, 1$, in order to satisfy the definition of the sone.

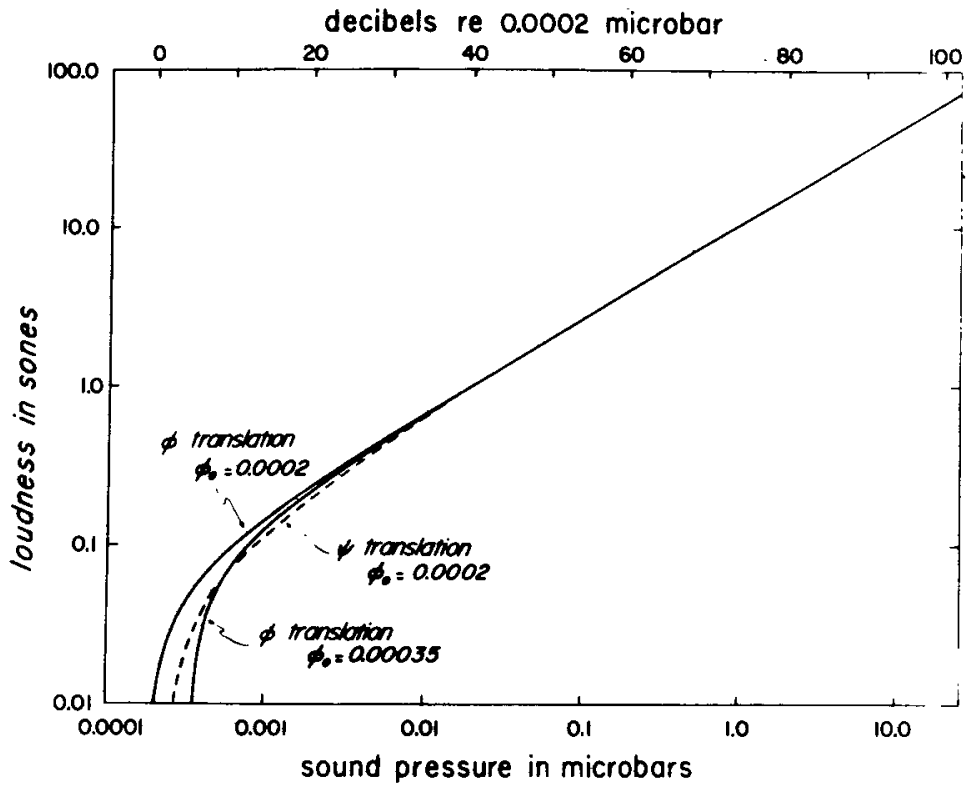

Fig. 2. The sone function (loudness as a function of sound presaure), plotted in accordance with two modifications of the psychophysical power law: the $\psi$ translation $\left(\varphi_{0}=0.0002\right.$ microbar $)$ and the $\varphi$ transhation $\left(\varphi_{0}=0.0002\right.$ microbar and $\varphi_{0}=0.00035$ microbar). 
The functions in Fig. 2 point up the fact that the departure of the loudness scale from a simple power function (Eq. 1) shows itself only over a minute fraction of the stimulus range and over a very small fraction of the subjective range; the logarithmic axes greatly exaggerate the apparent degree of departure. In this range (about 0 to $20 \mathrm{~dB}$ SPL) the sounds are extremely soft, judgments of apparent magnitude are highly variable, and the probabilistic nature of detection makes itself evident.

The difficulty of distinguishing between the $\psi$ and $\varphi$ versions might be easier if we knew exactly what the nature of the sensory threshold is and exactly what its "quantity" is. Actual threshold measures depend on the psychophysical method used, on the precise conditions of the experiment, and on the statistical definition adopted by the experimenter. The threshold must, therefore, always be defined in the context of its measurement, and in scaling experiments the use of suprathreshold stimuli will undoubtedly influence its exact location. Note that in Fig. 2, if $\varphi_{0}$ were known in advance to equal exactly 0.0002 microbar, the predicted difference belween the $\psi$ and $\varphi$ versions would amount to as much as $3 \mathrm{~dB}$ at the low ends of the functions. (In 1966 Fagot described a procedure that might be of use in distinguishing between the $\psi$ and $\varphi$ versions, given independent knowledge of the value of the absolute threshold.) In fact, however, the quantity $\varphi_{0}$ is not known a priori with precision. If $\varphi_{0}$ is estimated from scaling data it becomes virtually impossible to distinguish between the $\psi$ and $\varphi$ versions, because the distinction must be made solely with respect to the nature of the curvature of the two functions. How difficult this would be is brought out in Fig. 2 by replotting the $\varphi$ version under the assumption that $\varphi_{0}$ equals 0.00035 microbar, rather than 0.0002 microbar.

Figure 3 gives another example of the difficulty of deciding empirically between the $\psi$ and $\varphi$ translations. Estimations of apparent warmth (J. C. Stevens \& Marks, 1967) were fitted by power laws with both $\psi$ and $\varphi$ iranslations, using iterative least squares procedures. It is obvious that the $\psi$ translation and the $\varphi$ translation both provide excellent descriptions of the data. This is so even though the value of $\varphi_{0}$ is a sizeable fraction of even the highest levels of the stimulus used.

The issue of the appropriate form of the psychophysical power law cannot be resolved by appeal to tests of "goodness of fit" on the results of a few experiments. For one thing, the outcome of a given experiment is easily biased by choice of method. For example, the fractionation ("halving") procedure used by Stewart, Fagot, and Eskildsen (1967) was bound to favor the $\psi$ over the $\varphi$ translation. The reader is referred to a review by $S$. S. Stevens (1962) for the reasons why fractionation (halving) by itself would yield a misleading picture; indeed the author of the power law states that reliance on the halving method helps to explain his failure to discover the power law much earlier. A method that was effective in concealing the power relation does not recommend itself for the testing of alternative versions of the power law.

Students of psychophysical scaling have repeatedly noted the potentially disruptive effects inherent in certain psychophysical procedures. For example, although the effects attributable to the use of a standard stimulus have been noted time and again (see, for examples, S. S. Stevens, 1956; Hellman \& Zwislocki, 1961; Bernyer, 1962), they are often overlooked in the choice of experimental procedures. The effects of a standard are often large enough to twist the form of the obtained psychophysical function so as to favor one or another equation artifactually. For this and other reasons, the experiment by Fagot and Stewart probably falls short of a definitive determination of the nature of the brightness function near threshold.

In their experument, the task was to estimate the brightness of a test target as a fraction of a simultaneously viewed standard target, whose brightness was called 1000 . The standard was the brightest stimulus used in the experiment. This procedure would seem to be an unfortunate choice, partly because a potentially disruptive standard was used and partly because the stimulus arrangement promoted brightness contrast (inhibition). As evidence that contrast was a factor in the experiment we note that the measured threshold was considerably higher in the presence of the standard

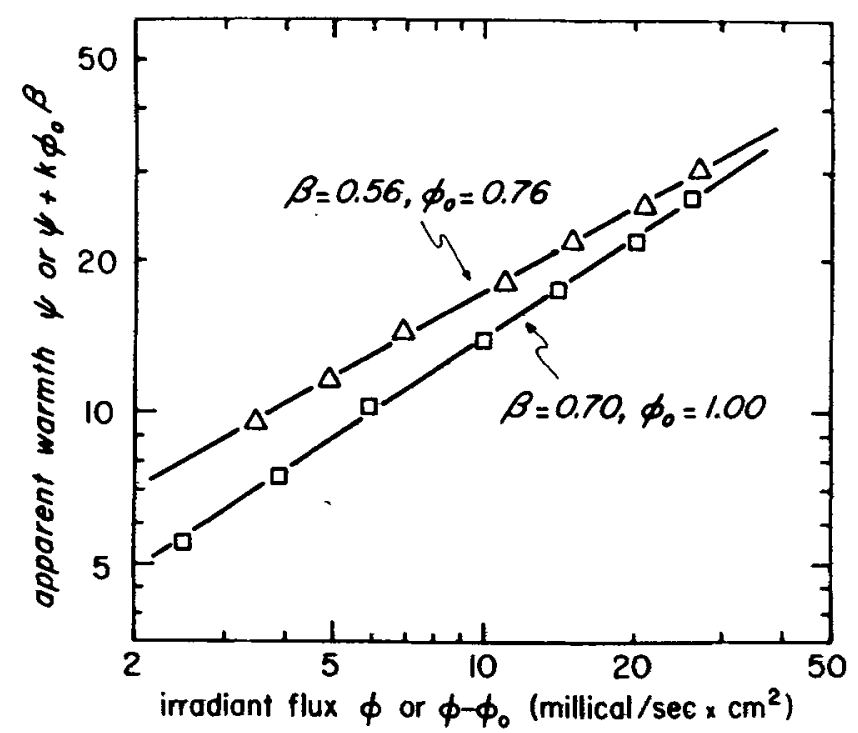

Fig. 3. Geometric mean estimates of apparent warmth as a function of irradiant flux in millical/sec $x \mathrm{~cm}^{2}$. The squares show warmth ( $\psi$ ) plotted as a function of $\varphi-1.0 \mathrm{millica} / \mathrm{sec} \times \mathrm{cm}^{2}$ ( $\varphi$ translation). The triangles show $\psi+k \cdot 0.76^{\beta}$ plotted as a function of $\varphi(\psi$ translation $)$.

than in its absence. The influence that contrast exerts on the form and slope of the brightness function (S. S. Stevens, 1966; S, S. Stevens \& Diamond, 1965) cannot be ignored in a study of this kind.

Even when pains are taken to keep the observer in a constant state of sensitivity and to avoid the known procedural constraints on the observer, the precision of the scaling techniques is probably not great enough to decide univocally between the $\psi$ and $\varphi$ translations, assuming one of them to be correct. For the time being, the issue may have to be dealt with in theoretical rather than empirical terms. As a practical matter, however, the issue is usually of little consequence. The similarity between the two translations means that either can be used to describe the empirical outcome with reasonable accuracy.

The logical difference between the $\psi$ translation and the $\varphi$ translation lies primarily in the interpretation of the absolute threshold. Proponents of the $\downarrow$ translation assert that the fundamental relation between apparent magnitude and stimulus magnitude is a simple power function (Eq. 1), and that the reduction in apparent magnitude to zero at the threshold results from masking by intrinsic physiological noise. In other words, sensitjvity of the sense organ is in the final analysis limited solely by the intrinsic noise level. This explanation may appeal in the case of sensory continua (e.g., loudness) for which absolute sensitivity is known to be great, and for which the intrinsic noise level might reasonably be expected to help determine the form of the psychophysical function.

On certain continua such as warmth, cold, and pain, absolute sensitivity is "poor," in that relatively large quantities of energy must be brought into play before a sensation is aroused. For example, irradiation of the skin over the range from 0 to 190 millical/sec $x \mathrm{~cm}^{2}$ (3-sec presentation) causes no pain. When this value is exceeded, however, pain appears and grows regularly as a power function of further increases in the irradiation up to at least $300 \mathrm{millical} / \mathrm{sec} \times \mathrm{cm}^{2}$, which feels very painful (Adair, J. C. Stevens, \& Marks, in press). The explanation that pain and irradiance are also related by a power function over the range between 0 and 190 millical $/ \mathrm{sec} x \mathrm{~cm}^{2}$ but that intrinsic noise masks pain up to 190 does not seem very plausible. The alternative view is that the pain grows as a power function of irradiance only after the threshold has been exceeded. It is of interest that this same type of reasoning has been used in biology to correct the power functions that describe the growth of individuals and 
populations as functions of time and body size (Angleton \& Pettus, 1966).

It appears to the authors that for many sensory continua modification of the power law by means of a $\varphi$ translation is called for. This is not to deny the possibility that in some instances intrinsic physiological noise may have an effect on the form of the psychophysical function near threshold. Even then, there is no a priori reason why the effect should be a simple subtraction from $\psi$ (Eq. 3). The effect of intrinsic physiological noise could be to subtract from $\varphi$ (Eq. 2) or to produce one of the other alternatives (Eqs. 4, 5, and 6) that have been proposed.

\section{REFERENCES}

ADAIR, E. R., STEVENS, J. C., \& MARKS, L. E. Thermally.induced pain, the dol scale, and the paychophysical power law. American Journal of Psychology, in press.

ANGLETON, G. M., PETTUS, D. Relative growth law with a threshold. Perspectives of Biological Medicine, 1966, 9, 421-424.

BERNYER, G. Etude sur la validité d'uné echelle de sensation d'effort musculaire. Année Psychologique, 1962, 62, 1-15.

EKMAN, G. Subjective power functions and the method of fractionation. Report from Psychology Laboratory, University of Stockholm, 1956, No. 34.

EKMAN, G. Methodological note on scales of gustatory intensity. Scandinavian Journal of Psychology, 1961, 2, 185-190.

EKMAN, G. The absolute threshold and the psychophysical function in brightness vision. Report from Psychology Laboratory, University of Stockholm, 1967, No. 233.

FAGOT, R. F. Alternative power laws for ratio scaling. Psychometrika, 1966, 31, 201.214.

FAGOT, R. F., \& STEWART, M. R. An experimental comprison of stimulus and response translated power functions. Perception \& Psychophysics, $1968,3,297.305$.

GALANTER, E., \& MESSICK, S. The relation between category and magnitude scales of loudness. Psychological Review, 1961, 68, 363-372.

HELLMAN, R. P., \& ZWISLOCKI, J. Some factors affecting the estimation of loudness. Journal of the Acoustical Society of America, 1961, 33, 687-694.

LOCHNER, J. P. A., \& BURGER, J. F. Form of the loudness function in the presence of masking noise. Journal of the Acoustical Society of America, $1961,33,1705-1707$.

LUCE, R. D. On the possible psychophysical laws. Psychological Review, $1959,66,81-95$.

McBURNEY, D. H. Magnitude estimation of the taste of sodium chloride after adaptation to sodium chloride. Journal of Experimental Psychology, $1966,72,869-873$.

MoGILL, W. A. The slope of the loudness function: A puzzle. In H. Gulliksen and S. Messick (Eds.), Psychological scaling: Theory and applications. New York: John Wiley, 1960. Pp. 67-81.

MOUNTCASTLE, V. B., POGGIO, G. F., \& WERNER, G. The relation of thalamic cell response to peripheral stimuli varied over an intensive continuum. Journal of Neurophysiology, 1963, 26, 807.834.

STEVENS, J. C., MARKS, L. E. Apparent warmth as a function of thermal irradiation. Perception \& Psychophysics, 1967, 2, 613-619.

STEVENS, J. C., \& STEVENS, S. S. Warmth and cold: Dynamics of sensory intensity. Journal of Experimental Psychology, 1960, 60, 183-192.

STEVENS, S. S. On the brighness of lights and the loudness of sounds. Science, 1953, 118, 576 (Abstract).

STEVENS, S. S. The direct estimation of sensory magnitudes-loudness. American Journal of Psychology, 1956, 69, 1-25.

STEVENS, S. S. Tactile vibration: Dynamics of sensory intensity. Journal of Experimental Psychology, 1959, 59, 210-218.

STEVENS, S. S. The surprising simplicity of sensory metrics. American Psychologist, 1962, 17, 29.39.

STEVENS, S. S. Power-group transformations under glare, masking and recruitment. Journal of the Acoustical Society of America, 1966, 39, 725.735 .

STEVENS, S. S., DIAMOND, A. L. Effect of glare angle on the brightness function for a small target. Vision Research, 1965, 5, 649-659.

STEVENS, S. S., GUIRAO, M. Loudness functions under inhibition. Perception \& Psychophysics, 1967, 2, 459-465.

STEWART, M. R., FAGOT, R. F., ESKILDSEN, P. R. Invariance tests for bisection and fractionation scaling. Perception Psychophysics, 1967, 2, 323.327.

ZWISLOCKI, J. Analysis of some auditory characteristics. In R. D. Luce, R. R. Bush, E. Galanter (Eds.), Handbook of mathematical psychology. Vol. III. New York: John Wiley, 1965. Pp. 1-97.

ZWISLOCKI, J., HELLMAN, R. On the "psychophysical law." Journal of the Acoustical Society of America, 1960, 32, 924 (Abstract).

\section{NOTES}

1. This study was supported by Contract F44620-67-C-0017 with the Air Force Office of Scientific Research.

2. Address: John B. Pierce Foundation Laboratory, 290 Congress Ave., New Haven, Conn. 06519 .

(Accepted for publication August 9, 1968.) 